\title{
Variables Significativas del Proceso de Combustión del Gas Natural
}

\author{
Gloria Villaflor $^{1}$, Graciela V. Morales ${ }^{2}$ y Jorge Velasco ${ }^{1}$ \\ Universidad Nacional de Salta, Facultad de Ingeniería, CIUNSa, (1) Instituto de Beneficio de \\ Minerales (INBEMI), (2) Instituto de Investigaciones para la Industria Química (INIQUI), \\ Avenida Bolivia 5150, 4400 Salta-Argentina \\ (e-mail: villaflo@unsa.edu.ar, gmorales@unsa.edu.ar, inbemi@unsa.edu.ar)
}

\begin{abstract}
Resumen
Se determinan las variables significativas del proceso de combustión de gas natural, aquellas más sensibles para producir cambios importantes desde punto de vista económico y medioambiental. Con este fin se realiza la simulación del proceso de combustión de gas natural, utilizando el simulador comercial HYSYS. Se determina que las variables de operación más sensibles para este proceso son la temperatura del aire, la temperatura de los gases de combustión y el exceso de aire usado en la combustión. Se concluye que el calor extraido disminuye a medida que aumenta la temperatura de los gases de combustión, que aumenta con el incremento de la temperatura del aire, y que el calor extraido disminuye con el aumento del exceso de aire, a temperatura de aire y de gases de combustión constantes.
\end{abstract}

Palabras clave: gas natural, combustión, simulación de procesos, medio ambiente

\section{Significant Variables in the Combustion Process of Natural Gas}

\begin{abstract}
The most significant operating variables of the natural gas combustion process, those most sensitive to produce economic and environmental impact, are determined. For this, simulation of the combustion process is carried out using the HYSYS commercial simulator. It is found that the most sensitive operating variables are the air temperature, the temperature of the combustion gases and the excess air used in the combustion. It is observed that the heat produced decreases as the combustion gases temperature increases, it increases as the air temperature increases, and that it decreases as the excess air increases at a constant temperature of the air and of the combustion gases.
\end{abstract}

Keywords: natural gas, combustion, process simulation, environment 


\section{INTRODUCIÓN}

La atmósfera es esencial para la vida sobre el planeta, sin embargo el hombre descarga en ella muchos de los residuos producidos por su actividad. Debido a la gran movilidad de la atmósfera los efectos de los residuos vertidos en ella pueden afectar extensas zonas, tanto a nivel local como regional y en algunos casos planetario o global. La contaminación del aire producida por la actividad del hombre, tiene su origen en causas muy diversas, siendo la combustión la principal. Los contaminantes producidos por la reacción de combustión son monóxido de carbono, óxidos de nitrógeno, dióxido de azufre, hidrocarburos y material particulado.

Se producen además grandes cantidades de dióxido de carbono como producto de combustión completa del carbono contenido en el gas natural. El dióxido no es considerado un contaminante, sin embargo la combustión de grandes cantidades de combustible fósiles aumenta su contenido en la atmósfera lo que puede originar un incremento de la temperatura global de la superficie del planeta conocido como efecto invernadero (Rao, 1991; Wark y Warner, 1997).

La importancia de realizar la simulación de procesos, como el de combustión, reside en el hecho de que, sin mayores costos económicos es posible realizar un estudio detallado del proceso con el objetivo de identificar las variables sensibles de operación, y determinar los valores a asignar a las mismas para mejorar el funcionamiento de un proceso ya existente o diseñar uno nuevo. Todo esto teniendo en cuenta tanto la rentabilidad del proceso como así también la preservación del medio ambiente. Veselý et al. (1996), simularon el proceso de combustión de gas natural determinando la temperatura y los perfiles de concentración de metano, oxígeno, monóxido y dióxido de carbono a la salida de la cámara de combustión. Encontraron que la perfomance de la cámara de combustión está fuertemente relacionada con la temperatura, la concentración de oxígeno y el tiempo de residencia del gas en la misma. Por otra parte, Khoshnoodi y Lim (1997), realizaron la simulación de la primera etapa de la producción de gas de síntesis a partir de gas natural. Utilizaron una herramienta de análisis de sensibilidad paramétrica que les permitió encontrar la temperatura óptima del reactor para maximizar la producción de CO en el gas de síntesis. Weber et al. (2000), simularon una cámara de combustión a altas temperaturas y grandes cantidades de gas. Observaron que el proceso de combustión fue mucho más lento que el proceso tradicional. Observaron, también, la presencia de flujos de calor radiactivo en alta proporción. Requirieron de un modelo químico simplicado para estas nuevas condiciones de combustión. Sonibare y Akeredolu (2004), simularon la combustión de gas natural de Nigeria, utilizando un modelo teórico basado en las ecuaciones de balance de materia, determinaron cuáles son las condiciones operativas que favorecen la combustión completa y cuáles llevan a la combustión incompleta. También, analizaron el efecto de impurezas en el gas natural que llegan a cambiar las condiciones anteriormente determinadas. Westbrook et al. (2005), realizaron una revisión sobre los progresos realizados en los últimos 50 años en el campo de la computación aplicada al proceso de combustión, prestando atención particularmente a los distintos modelos desarrollodos para fluidodinámica y cinética química.

En este trabajo se realiza la simulación del proceso de combustión del gas natural con el fin de determinar las variables de operación sensibles para producir cambios importantes desde punto de vista económico, preservando el medio ambiente (Chomiack,1990).

\section{SIMULACION DEL PROCESO}

\section{Composición del Gas Natural}

En la Tabla 1 se indica la composición del gas natural utilizado para la simulación del proceso de combustión.

Tabla 1: Composición del Gas Natural

\begin{tabular}{|l|c|l|l|l|c|}
\hline Composición & $\mathrm{CH}_{4}$ & $\mathrm{C}_{2} \mathrm{H}_{6}$ & $\mathrm{C}_{3} \mathrm{H}_{8}$ & $\mathrm{CO}_{2}$ & $\mathrm{~N}_{2}$ \\
\hline \% Volumen & 90,87 & 6,19 & 0,27 & 1,88 & 0,79 \\
\hline
\end{tabular}




\section{Reacciones de Combustión del gas natural}

Se propone el sistema de reacciones linealmente independientes siguiente, que se llevan a cabo en la cámara de combustión:

$$
\begin{gathered}
\mathrm{CH}_{4}+2 \mathrm{O}_{2} \rightarrow \mathrm{CO}_{2}+2 \mathrm{H}_{2} \mathrm{O} \\
2 \mathrm{CH}_{4}+3 \mathrm{O}_{2} \rightarrow 2 \mathrm{CO}+4 \mathrm{H}_{2} \mathrm{O} \\
2 \mathrm{C}_{2} \mathrm{H}_{6}+7 \mathrm{O}_{2} \rightarrow 4 \mathrm{CO}_{2}+6 \mathrm{H}_{2} \mathrm{O} \\
\mathrm{C}_{3} \mathrm{H}_{8}+5 \mathrm{O}_{2} \rightarrow 3 \mathrm{CO}_{2}+4 \mathrm{H}_{2} \mathrm{O}
\end{gathered}
$$

El nitrógeno contenido en el gas natural se comporta como inerte en el proceso de combustión, ya que la formación de óxidos de nitrógeno no afecta la cantidad de aire necesario para la combustión ni la cantidad de gases de combustión producidos. Sin embargo desde el punto de vista ambiental es importante considerar la formación de dichos óxidos por la contaminación que producen los mismos.

Dado que para las reacciones de combustión la constante de equilibrio es sumamente grande, pueden considerarse a las reacciones (1) a (4) como irreversibles y de primer orden; considerando que los modelos cinéticos encontrados en la bibliografía para reacciones de combustión, corresponden en su mayoría a cinéticas de primer orden (Szekely et al., 1976; Levenspiel, 1987).

\section{Simulador HYSYS}

La simulación del proceso de combustión se realizó utilizando el simulador de procesos HYSYS. Para el cálculo de las propiedades fisicoquímicas de las corrientes de gas natural y de aire a distintas condiciones de presión y temperatura, se seleccionó a la ecuación de Peng Robinson como ecuación de estado.

La estructura básica que se aplica en la simulación de proceso usando Hysys consiste en tres etapas: 1) formulación del problema; 2) simulación del proceso (cálculo); y 3) análisis de resultados.

En este trabajo se asimiló la cámara de combustión a un reactor tipo "batch", ya que el simulador utilizado no dispone del módulo de combustión. En la Fig. 1 se muestra el esquema utilizado para la simulación. Los hidrocarburos (corriente: Gas) y el aire (corriente: Aire) son combinados en un mezclador y la corriente mezclada (Mezcla) es alimentada al reactor que opera a presión atmosférica, siendo nula la corriente líquida de salida del reactor (Líq).

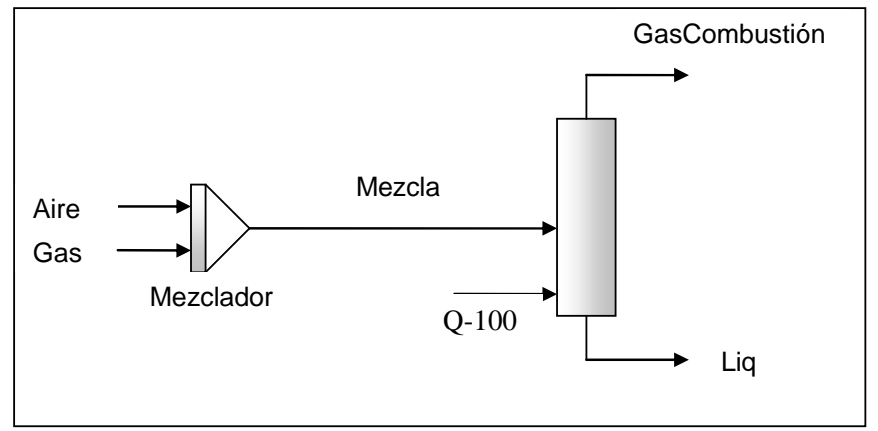

Fig. 1: Esquema de la simulación del Proceso de combustión

Se utilizó el "reactor de conversión" disponible en el simulador. El reactor de conversión es un tanque agitado discontinuo en el cual ocurren reacciones de conversión; siendo las mismas, reacciones que 
proceden hasta que se alcanza una conversión especificada o hasta que desaparece el reactivo limitante (HYSYS, 2003). El hecho de trabajar con reacciones de conversión no hace necesario el uso de expresiones cinéticas. El programa de simulación calcula el porcentaje de conversión alcanzado como:

$X(\%)=\frac{\mathrm{N}_{\mathrm{Ae}^{-N}} \text { As }}{\mathrm{N}_{\text {Ae }}} \times 100$

Siendo: $\mathrm{N}_{\mathrm{Ae}}$ el flujo de entrada al reactor del componente guía, $\mathrm{A}$.

$\mathrm{N}_{\text {As }}$ el flujo de salida del reactor del componente guía, $\mathrm{A}$.

Los resultados de simulación verificaron los balances de materia y energía en el reactor.

\section{RESULTADOS Y DISCUSION}

En la Tabla 2 se presentan algunas propiedades, calculadas por el simulador, de la corriente mezcla gas natural-aire que entra a la cámara de combustión a $25^{\circ} \mathrm{C}$, y de los gases de combustión producidos a $300^{\circ} \mathrm{C}$.

Tabla 2: Propiedades de mezcla gas natural-aire y de los gases de combustión

\begin{tabular}{|l|c|c|}
\hline & Mezcla gaseosa & Gases de combustión \\
\hline Peso Molecular & 27,81 & 27,72 \\
\hline Densidad $\left(\mathrm{kg} / \mathrm{m}^{3}\right)$ & 1,138 & 0,5895 \\
\hline Capacidad Calorífica $\left(\mathrm{kJ} / \mathrm{kg}^{\circ} \mathrm{C}\right)$ & 1,077 & 1,195 \\
\hline Poder calorífico inferior $(\mathrm{kJ} / \mathrm{kg})$ & 2736 & $2,424 \times 10^{-5}$ \\
\hline $\mathrm{Cp} / \mathrm{Cv}$ & 1,391 & 1,338 \\
\hline $\mathrm{Cv}\left(\mathrm{kJ} / \mathrm{kg}^{\circ} \mathrm{C}\right)$ & 0,7742 & 0,8929 \\
\hline Viscosidad $(\mathrm{cP})$ & 0,001784 & 0,002655 \\
\hline Conductividad calorífica $(\mathrm{W} / \mathrm{m} \mathrm{K})$ & 0,002644 & 0,004254 \\
\hline
\end{tabular}

En la Fig. 2 se muestran las fracciones molares de los gases de combustión producidos en función del coeficiente de exceso del aire utilizado, n, y considerando una temperatura de entrada de aire de $25^{\circ} \mathrm{C}$. Se trabajó variando el exceso de aire desde $n=1$, mínimo o estequiométrico, hasta $n=1,20$, rango de coeficientes de exceso normalmente utilizado para combustión de gas natural. El cálculo de los flujos de aire estequiómetrico y el exceso se realizó utilizando un programa desarrollado en Planilla de Cálculo Excel 2000 (Villaflor et al., 2005; Pocoví et al., 2003). Se observa la disminución de las fracciones molares del agua y del dióxido de carbono con el exceso de aire utilizado, debido a que el exceso de aire diluye los gases de combustión.

Por otra parte en la Fig. 3 se muestra el efecto de la temperatura de los gases de combustión sobre el calor extraido (calor entregado a la carga), tomando una temperatura del aire de $25^{\circ} \mathrm{C}$, utilizando distintos excesos de aire. Se observa que a medida que aumenta la temperatura de los gases de chimenea disminuye el calor extraido, porque aumenta el calor sensible perdido en los gases al aumentar la temperatura de los mismos. Se observa, también, que a temperatura de gases de chimenea constante el calor extraído disminuye con el exceso de aire. 


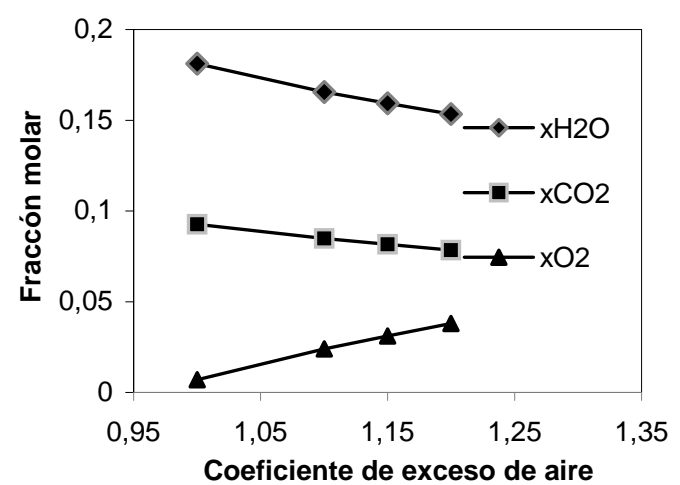

Fig. 2: Efecto del exceso de aire en la composición de los gases de combustión

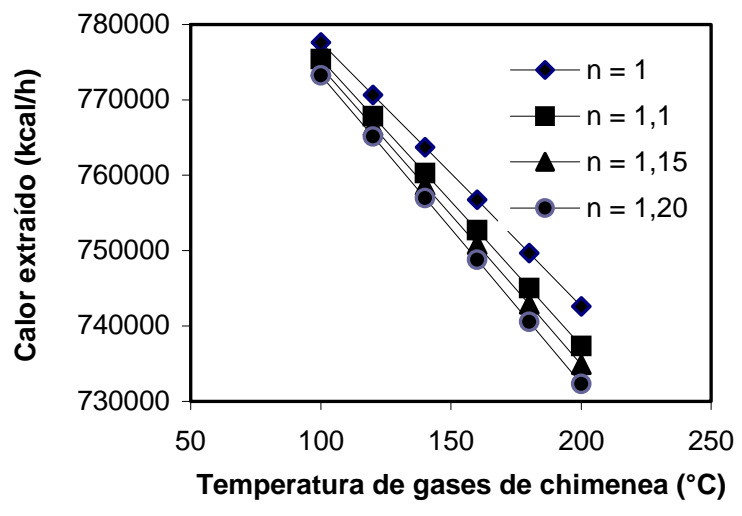

Fig. 3: Efecto de la temperatura de gases de combustión sobre el calor extraido

En la Fig. 4 se presenta el efecto de la temperatura de precalentamiento del aire sobre el calor extraido, manteniendo constante la temperatura de gases de combustión $\left(300{ }^{\circ} \mathrm{C}\right)$, utilizando distintos excesos de aire. Se observa el aumento del calor extraido con el incremento de la temperatura de precalentamiento del aire debido a la recuperación parcial del calor contenido en los gases de combustión y además la disminución del calor extraído con el aumento del exceso de aire.

En la Fig. 5 se presentan los efectos de los calores extraidos o entregados a la carga y perdidos con los gases de combustión en función del exceso de aire, manteniendo la temperatura de los gases de combustión constante $\left(120^{\circ} \mathrm{C}\right)$. Se observa que el calor perdido en los gases de combustión aumenta con el exceso de aire, porque aumenta el calor sensible al ser mayor la cantidad de gases de combustión, mientras que el calor extraído disminuye con el mismo porque se tiene que calentar mayor cantidad de aire sin que éste aporte calor.

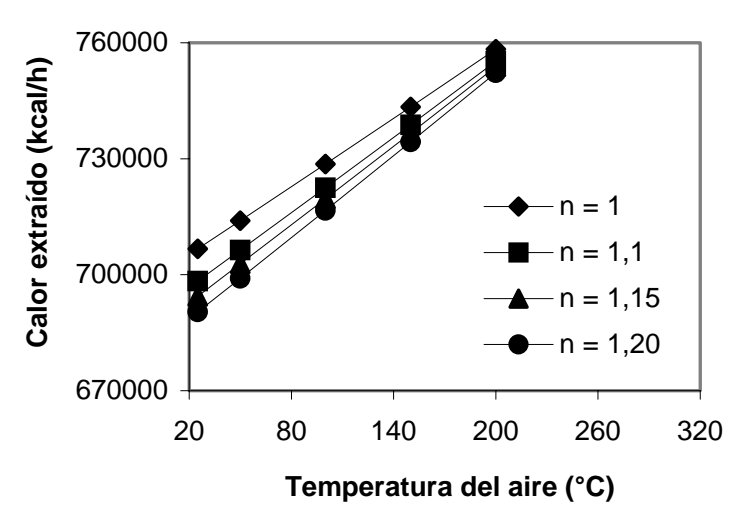

Fig. 4: Efecto de la temperatura del aire sobre el calor extraído

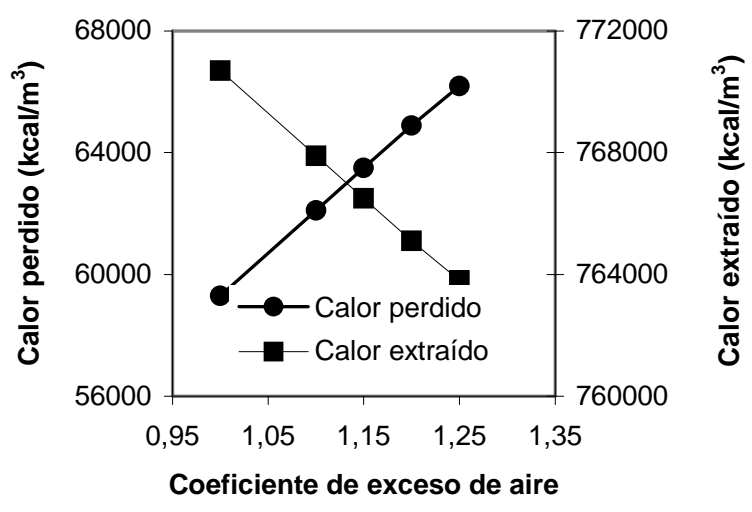

Fig. 5: Efecto del exceso de aire sobre el calor perdido y el calor extraído

\section{CONCLUSIONES}

El resultado de la simulación del proceso de combustión de gas natural permite concluir que el exceso de aire a utilizar debe ser el mínimo posible, compatible con la combustión completa del combustible, ya que el mismo diluye los gases de combustión y consume calor. Este calor perdido aumenta el consumo de combustible, por lo que el uso de exceso de aire correcto conlleva un ahorro de combustible, con la consiguiente disminución de emisión de dióxido de carbono a la atmósfera.

Además conviene aprovechar el calor de los gases de combustión, para precalentar el aire, disminuyendo la temperatura de salida de los gases de chimenea, teniendo en cuenta que ésta debe 
ser superior a la temperatura de rocío del vapor de agua contenido en los mismos, de este modo se evita la condensación que puede originar serios problemas de corrosión.

En consecuencia se determina que las variables significativas del proceso de combustión de gas natural son: Exceso de aire (mínimo compatible con la combustión completa del combustible), temperatura del aire (con precalentamiento utilizando el calor sensible de los gases de chimenea) y temperatura de los gases de salida de chimenea (superior a la temperatura de rocío del vapor de agua contenido en los mismos).

Desde el punto de vista del uso del simulador de procesos se puede concluir que es una herramienta útil que permite calcular las propiedades fisicoquímicas de las corrientes gaseosas, proponer distintos esquemas del proceso y probar diferentes condiciones operativas.

El trabajo realizado resulta de inmediata aplicación tanto en la enseñanza del tema de combustión de gas natural como para equipos que trabajan en el diseño de procesos de combustión o de mejoras de un proceso en operación, desde el punto de vista económico y de preservación del medio ambiente.

\section{REFERENCIAS}

Chomiack, J.; Combustión, a study in theory, fact and application, Energy \& Engineering Science (1990).

HYSYS v3.2, Aspen Tecnology Inc., Hyprotech Technical Suport. Hyprotech, Canadá (2003).

Khoshnoodi M. y Y. S. Lim; Simulation of partial oxidation of natural gas to synthesis gas using ASPEN PLUS, Fuel Processing Technology: 50(2-3), 275-289 (1997).

Levenspiel, O.; El Minilibro de los Reactores Químicos, Editorial Reverté, Barcelona (1987).

Pocoví, R., G. Villaflor, J. Velasco y E. Bisonard; Servicios Auxiliares, Ed. Magna (2003).

Rao C.S.; Environmental Pollution Control Engineering, J. Willey \& Sons, New York, USA (1991).

Sonibare, J.A. y F. A. Akeredolu; A theoretical prediction of non-methane gaseous emissions from natural gas combustion, Energy Policy: 32(14), 1653-1665 (2004).

Szekely, J., J.W. Evans y H.Y. Sohn; Gas - Solid Reactions, Academic Press, New York (1976).

Villaflor G., J. Velasco, R. Pocoví y E. Bisonard; Combustión y evaluación de las emisiones producidas por combustión, Actas del VII CAIP, p.105-108, Vila Real, Portugal, Setiembre (2005).

Veselý V., H. Miloslav, O. Trnka y D. Fetsch; Performance of an afterburner chamber with a natural gas burner, Fuel: 75(11), 1271-1273 (1996).

Wark K. y C. Warner; Contaminación del aire. Origen y control, Ed. Limusa (1997).

Weber R., S. Orsino, N. Lallemant y A.Verlaan; Combustion of natural gas with high-temperature air and large quantities of flue gas, Symposium (International) on Combustion: 28(1), 1315-1321 (2000).

Westbrook C.K. y otros cuatro autores; Computational combustion, Proceedings of the Combustion Institute: 30(1), 125-157 (2005). 